\title{
Effect of Noise Type and Signal-to-Noise Ratio on the Effectiveness of Noise Reduction Algorithm in Hearing Aids: An Acoustical Perspective
}

\author{
Sharath Kumar KS* and Manjula P \\ Department of Audiology, All India Institute of Speech and Hearing (AIISH), University of Mysuru, India
}

Submission: March 05, 2016; Published: March 16, 2017

*Corresponding author: Sharath Kumar KS, Department of Audiology (New JC block), Manasagangotri, AIISH, Mysuru - 570 006, Karnataka, India, Tel: +919845421986; Email: sharathkumarks08@ gmail.com

Second author: Manjula P, Professor of Audiology, Department of Audiology, Manasagangotri, AIISH, Mysuru - 570006 India, Karnataka, Tel: +919731251948; Email: manjulap21@hotmail.com

\begin{abstract}
Purpose: The study evaluated factors that influence the effectiveness of noise reduction (NR) algorithm in hearing aids, through acoustical measures.

Methods: Factorial design was employed. Study sample: The output from hearing aid, with and without NR at three signal-to-noise ratios (SNR) with five types of noise, was recorded. The effect of noise reduction was studied through objective measures such as Waveform Amplitude Distribution Analysis - Signal-to-Noise Ratio (WADA-SNR), Envelope Difference Index (EDI), and Perceptual Evaluation of Speech Quality (PESQ).

Results: The results revealed that when NR was enabled, it was effective in reducing the noise. However, when speech was presented in the presence of noise, the NR was effective in enhancing certain acoustic parameters of speech; like signal-to-noise ratio and envelope.

Conclusion: The study utilized group of parameters in assessing the output from the hearing aid which helped in elaborating the subtle changes in the output of the hearing aid with and without NR processing. NR algorithm when 'ON' is helpful in reducing noise which in turn can improve speech perception and thus the duration of hearing aid usage. However, the amount of reduction provided by NR algorithm depends on the type of noise, and the SNR in the environment.
\end{abstract}

Keywords: Acoustic analysis; EDI; Hearing aid; Noise reduction algorithm; PESQ; Signal-to- noise ratio; WADA-SNR

Abbreviations: NR: Noise Reduction; SNR: Signal-to-noise ratio; WADA-SNR: Waveform Amplitude Distribution Analysis-Signal to Noise Ratio; EDI: Envelope Difference Index; PESQ: Perceptual Evaluation of Speech Quality; ANL: Acceptable Noise Level; DNR: Digital Noise Reduction; KEMAR: Knowles Electronic Manikin for Acoustic Research; ANOVA: Analysis of Variance

\section{Introduction}

Hearing aids are amplification devices recommended for persons with hearing impairment. Over the years, due to advancements in research and digitization, hearing aids have undergone immense changes in technology. Implementation of technologies such as directional microphones, compression and noise reduction algorithms in hearing aids have improved perception of amplified speech. In literature, the benefit from directional microphones, wide dynamic range compression, noise reduction (NR) algorithms in hearing aids are assessed using behavioral measures, such as speech tests in quiet and in the presence of noise, and by using subjective rating scales [1-4].
The hearing aid processing has been studied by analyzing the output of the hearing aid with varying the type of compression, compression parameters, omni-directional vs. directional microphones and noise reduction algorithms [5-10]. The benefit from the NR algorithms can be evaluated in two ways. One is to study the amount of reduction in noise when there is noise alone; and second is to evaluate the perception of speech in the presence noise. The amount of noise reduced by NR algorithm in noise alone conditions / environment is important since comfort in noisy condition is also a factor in determining the overall satisfaction in a hearing aid user, which in turn affects the duration of hearing aid use. 
The advanced signal processing algorithms within the hearing aid have found to affect the acoustics of the signal and also the quality of the signal $[11,12]$. In behavioral measures, the effect of hearing aid processed sound perception is evaluated by varying types of noise, signal to noise ratio, and many other parameters. In addition to this, client-related factors such as age, etiology of hearing loss, type, configuration of hearing loss and cognitive factors also play a role in perception of hearing aid processed speech. In order to assess the hearing aid processing alone, it is required to exclude these client-related factors. Studying the output from the hearing aid objectively through acoustical measures will help in understanding the changes occurring on the input signal due to digital signal processing [13]. It will also throw light on understanding the instrument functioning and working principle of different features of the hearing aid. In addition, these objective measures will aid in development of better signal processing strategies and algorithms by providing feedback on present strategies and algorithms.

The acceptable noise level (ANL) is one of the behavioral means used in the recent years to measure listening comfort in noise. The ANL is the maximum level of background noise that an individual is willing to accept while listening to speech. Research has shown that the use of directional microphone and NR algorithm can reduce the aided ANLs [14]. A study on the effect of digital noise reduction (DNR) on perception of aversive and annoying sounds using the ANL measure showed that the DNR has a positive impact by reducing the annoyance caused by traffic noise [15]. The authors concluded that it was valuable recommending the DNR feature for a person using a hearing aid. Thus, it becomes important also to evaluate the amount of noise reduced by NR algorithm. This can help in understanding the amount of noise that a NR algorithm is able to reduce; which in turn could increase the comfort in noise. This can be evaluated by presenting only noise and assessing the hearing aid output. This can be correlated to the amount of improvement in ANL, behaviorally.

The acoustical effect of the noise reduction in the hearing aid has been studied in the past [7,12,16-19]. In addition, literature also reveals that the acoustic measures of the hearing aid output have been studied for hearing aid parameters such as compression, noise reduction algorithms or with a combination of both $[6-8,12,13,20,21]$. However, there were many technical difficulties faced in separating the speech from noise. One of the techniques to study acoustical parameters of a hearing aid included a simple measure like estimation of signal-to-noise ratio [19]. Though the procedure was simple and basic, they had good predictive abilities [22]. In the due course of technical advancements, more computational models for measuring the acoustic output have emerged. Many MATLAB (Matrix Laboratory) based codes have emerged to assess the acoustical aspects of hearing aid output to improve the predictability of the signal-to-noise ratio (SNR), the envelope of the processed signal and also the overall quality difference between the processed and unprocessed signal.

One among them being Waveform Amplitude Distribution Analysis - Signal-to-Noise Ratio (WADA-SNR) [23], that evaluated the performance of the Waveform Amplitude Distribution Analysis - Signal-to-Noise Ratio (WADA-SNR) algorithm, (an MATLAB based application) on signals corrupted by white noise, background music, and interfering speech. They reported that the WADA-SNR algorithm showed less bias and less variability with respect to the type of noise compared to the other standard SNR estimation methods. As SNR methods have good predictive abilities, this method was used in the present study to analyse the output from the hearing aid. The Envelope Detection Index (EDI), one of the MATLAB based methods to analyze the temporal properties of a signal, has gained popularity because it can be compared directly with the subjective performance [24]. The EDI offers a simple and convenient way to capture temporal distortion and to characterize temporal envelope change while studying various other effects [24]. Hence in the present study, EDI was considered as a temporal measure to assess the hearing aid output.

In addition, models based on loudness e.g. [25], estimation of excitation patterns e.g. [26] and many approaches based on psychoacoustic models e.g. [27] were developed. Some of the objective measures used multiple components to capture various aspects of signal quality to collectively improve the overall prediction [22]. One of such models is the Perceptual Evaluation of Speech Quality (PESQ) [28-30]. It has been shown in the literature that measures such as frequency weighted segmental SNR (fwseg SNR) and perceptual evaluation of speech quality (PESQ) have both been shown to predict perceived speech quality [19,31]. A few studies [31,32] have tested the PESQ measure in predicting speech intelligibility. High correlation ( $\mathrm{r}$ $=0.9 ; \mathrm{r}=0.77-0.79$ ) was reported for predicting intelligibility of consonants and sentences in noise. Hence, PESQ measure was considered in the present study to analyze the amount of quality change in the presence of different types of noise and SNRs. In the recent years, Kates and Arehart [33] developed the Hearing Aid Speech Quality Index (HASQI) which is specifically designed for individuals with hearing impairment and for hearing aid applications. The HASQI is an objective measure of quality, designed and validated to predict subjective quality for distorted speech [33].

Research studies focusing on the effectiveness of NR algorithm alone through acoustical measures would give directions for advancements to be made in the algorithms to improve the performance. In order to ascertain the effectiveness of NR algorithm on different types of noise and at different SNRs, five types of noise encountered in everyday situations and three SNRs ( $+5 \mathrm{~dB}, 0 \mathrm{~dB}$ and $-5 \mathrm{~dB}$ SNRs) have been evaluated in the present study. Further, objective measures such as SNR estimation, temporal changes through WADA-SNR and EDI, 
and quality assessment through PESQ, have been employed in the present study to enumerate the amount of noise reduction. These measures tap different acoustical aspects which would bring about changes in perception of a speech signal. Thus, the aim of the present study was to analyze the acoustic changes brought about by NR algorithm in the hearing aid. This was verified by studying different variables such as different types of noise and SNRs, which could provide useful clues regarding the changes that are perceptible to listeners.

\section{Materials and Methods}

The aim of the study was to evaluate the variables that would influence the effectiveness of noise reduction (NR) algorithm in hearing aids, through acoustical measures. The following method was adopted to study this.

\section{Stimuli}

One list containing ten recorded sentences from Kannada sentence lists developed by Geetha et al. [34] was used to study the effectiveness of NR algorithm in hearing aid. The types of noise included were speech babble (eight-talker speech babble recorded by Anitha \& Manjula [35]), fan noise, traffic noise, cafeteria noise, and white noise. The traffic noise, fan noise and cafeteria noise were recorded using a Sound Level Meter (SLM; Bruel \& Kjær Type 2270). The pre-polarized free-field microphone of the SLM was held approximately at one meter distance from the sound source. The traffic noise was recorded during the moderate traffic flow on a busy road of Mysuru city. Fan noise was recorded with a ceiling fan running at a moderate speed (speed ' 3 ' of the ' 5 ' levels on the fan regulator). Cafeteria noise was recorded in a busy café during the moderate crowd hours. Each noise sample was recorded for duration of 30 minutes. The recorded noises were then transferred to a personal computer and were edited and played via Adobe Audition software 3.0. The white noise was generated using the Adobe Audition software 3.0.

\section{Procedure}

A commercially available digital behind-the-ear (BTE) hearing aid with a fitting range from mild to moderately-severe degree of hearing loss was selected. The selected hearing aid had eight channels with multi-channel noise reduction algorithm. This algorithm utilizes a modulation based noise detection and channel-wise noise reduction facility. The hearing aid was programmed through NOAH Link, using the NOAH software (version 4) and the hearing aid specific software that were installed in the personal computer. The audiogram was plotted in the NOAH software. The audiogram was simulated to mimic a sensorineural hearing loss with thresholds being $50 \mathrm{~dB}$ HL (both air-conduction or AC \& bone-conduction or BC) at all audiometric frequencies. The hearing aid was programmed using the NALNL2 prescriptive formula. The hearing aid programming was set an acclimatization level appropriate to a new hearing aid user at the time of programming. Thus, at the time of programming the hearing aid, the 'first fit' settings was applied. The target gain of the hearing aid ranged between $15 \mathrm{~dB}$ and $24 \mathrm{~dB}$ across the frequencies. The compression in the hearing aid was disabled. This was verified by performing an electroacoustic measurement of the programmed hearing aid. The microphone was set to omni-directional mode. This programmed BTE was considered for recording the output of the hearing aid when fitted on the ear of theKnowles Electronic Manikin for Acoustic Research (KEMAR).

The output from the hearing aid was recorded in the three conditions. They were:

a) Speech Alone Condition: Ten sentences presented at $65 \mathrm{~dB}$ SPL through a personal computer routed via Lynx Sound card and were recorded in the KEMAR microphone, without the hearing aid. The level was monitored using an SLM. This served as the reference 'unprocessed speech' for comparison with the two aided conditions.

b) Noise Alone Condition: Cafeteria noise, fan noise, speech babble, traffic noise and white noise were given individually as input to the hearing aid fitted to the KEMAR, at $65 \mathrm{~dB}$ A (LAeq). The hearing aid output at the ear of the KEMAR was recorded with NR algorithm of the hearing aid in 'ON' and 'OFF' conditions.

c) Speech in Noise Condition: The recorded sentence was presented through one loudspeaker kept at $0^{\circ}$ Azimuth at one meter distance from the KEMAR; and noise through the second loudspeaker, also kept at $0^{\circ}$ Azimuth at one meter distance from the KEMAR, simultaneously. The noise was presented for 15 seconds before the onset of speech in order to activate the noise reduction algorithm in the hearing aid. The noise levels were varied to get different input signal to noise ratios $(+5,0$ and -5 $\mathrm{dB}$ ) and the speech input level was kept constant at $65 \mathrm{~dB}(\mathrm{~A})$. This was repeated for each of the different types of noise. The output of the hearing aid, with NR algorithm 'ON' and 'OFF', in the ear of the KEMAR was recorded for analysis.

\section{Instrumental set-up for recording the output from the hearing aid.}

The G.R.A.S. 45BB KEMAR with the ear simulator RA0045 was placed at the center of the test room. The programmed BTE hearing aid was placed on the pinna of the KEMAR and the hearing aid was connected to the canal with an ear mold simulator. The recorded signal were presented through a personal computer routed via Lynx Sound card (Lynx AES 16 card, two Aurora 16 A to D convertor) and presented through two loudspeakers were mounted in front, adjacent to each other. The loudspeakers were at a distance of one meter from the KEMAR. The two loudspeakers were calibrated such that the output reaching the ear of KEMAR was same from both the loudspeakers. The speech was always presented through first loudspeaker and noise was always presented through the second loudspeaker. Block diagram 3 illustrates the block diagram of the instrumental set-up. 
The output from the hearing aid was picked up using $1 / 2$ inch microphone (Type 40AG) located in the KEMAR, monitored, recorded, and stored using the sound analyzer (Brüel \& Kjær Type 2270) (Figure 1). The level of the input signal was also monitored through Brüel \& Kjær 2270 sound analyzer during the recording process.

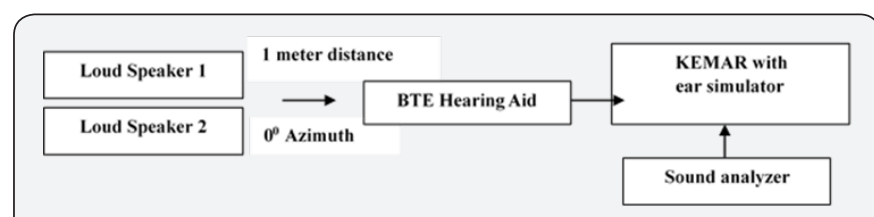

Figure 1: Block diagram of the instrumental set-up for recording the hearing aid output.

\section{Analysis}

The hearing aid output was measured in three stimuli conditions. They are given below:

a) Speech alone condition: The recorded output from KEMAR microphone for ten sentences without hearing aid served as 'unprocessed' speech. This was considered as reference to be compared with recorded output from the hearing aid with NR algorithm 'ON' and NR algorithm 'OFF' settings.

b) Noise alone condition: The hearing aid output for the cafeteria noise, fan noise, speech babble, traffic noise and white noise were analysed using the virtual Sound Level Meter (SLM), a MATLAB code developed by Lanman [36]. Each type of noise was presented for 60 seconds. This code is based on graphic user interface. Total LAeq in $\mathrm{dB}(\mathrm{A})$ and LAeq at 90 th percentile were calculated for each type of noise. This is the sound pressure level of noise level that exceeded for $90 \%$ of the measurement time. i.e., for $90 \%$ of the time, the noise level is above this measured level. The analysis of hearing aid output in the noise alone condition, with NR algorithm 'ON' and 'OFF' setting, was done for the middle 40 seconds duration out of the total 60 seconds of stimulus duration. It was evident from the spectral plot of the noise with the NR algorithm 'ON' condition that the NR algorithm was activated within 10 seconds of the stimulus onset. Hence, the duration was decided based on the spectral analysis of the NR processed output in noise alone condition. The difference between the LAeq values with NR algorithm 'ON' and NR algorithm 'OFF' provided the amount of reduction of noise brought about by the NR algorithm of a hearing aid. The value of overall LAeq and at 90th percentile in $\mathrm{dB}(\mathrm{A})$ were analysed and tabulated for NR algorithm 'ON' and NR algorithm 'OFF' conditions. The difference of LAeq values between NR algorithm 'ON' and NR algorithm 'OFF' was also computed and tabulated. This was done for each type of noise.

It is well documented that speech perception in noise depends on many factors. Of those factors, the overall signal envelope preserved in the presence of noise, SNR and the acoustic quality of speech in the presence of noise play role in perception of the speech signal [12]. To tap these features of speech, the WADA-SNR, EDI and PESQ were estimated.
Waveform Amplitude Distribution Analysis (WADA), given by Kim and Stern [23], was used to estimate the SNR, henceforth referred to as WADA-SNR. The measurement procedure was incorporated in a MATLAB code developed by Ellis [37], version 0.3 . This code automatically calculates the SNR in $\mathrm{dB}$ by comparing the sentences recorded in the presence of noise, while the NR algorithm was enabled and disabled.

Perceptual Evaluation of Speech Quality (PESQ) is an objective method for predicting the subjective quality of speech. This was developed by the International Telephone Union (ITU) for assessing the sound quality in telephone systems. The PESQ predicts how close the target signal is in terms of its quality, in comparison to the original signal. In the present study, the comparison has been done between speech in noise with NR algorithm 'ON' and the unprocessed speech. The comparison was done also for speech in noise with NR algorithm 'OFF' and the unprocessed speech. The PESQ provides a value between 0 and 4.5. If the value is 4.5 , then the target speech is same as unprocessed speech in terms of quality. This measurement procedure was incorporated in a MATLAB code developed by Ellis [37], version 0.3. This code automatically provided the mean opinion scores (MOS) for PESQ.

Envelope Difference Index (EDI) is a non-standardized MATLAB code based on the method given by Fortune et al. [38] was used to calculate the EDI. The EDI, being a temporal measure, was used to evaluate how close the two signals are in their envelope. The EDI values range from 0 to 1 . The value of 0 indicates perfectly similar envelopes and the value of ' 1 ' indicates completely dissimilar envelopes. The envelopes between speech in noise with $\mathrm{NR}$ algorithm 'ON' condition and the unprocessed speech; and speech in noise with NR algorithm 'OFF' and the unprocessed speech were compared in order to obtain the EDI.

The above parameters were assessed for all the ten sentences in the presence of each of the five types of noise viz., cafeteria noise, fan noise, speech babble, traffic noise and white noise; that were recorded from the hearing aid at three input SNRs viz., $-5,0$ and $+5 \mathrm{~dB}$. This gave rise to a total of thirty conditions for analyses viz., five types of noise $x$ three input SNRs $x$ two NR algorithm settings.

\section{Results}

The results have been given under noise alone condition and speech in noise conditions.

\section{Noise Alone Condition}

The hearing aid output for noise alone condition for five types of noise (cafeteria, fan, speech babble, traffic and white noise) in terms of mean value of overall LAeq and LAeq at 90th percentile in $\mathrm{dB}(\mathrm{A})$, with NR algorithm 'ON' and NR algorithm 'OFF', were tabulated and subjected to statistical analyses. The mean values of overall LAeq and 90th percentile LAeq for different types of noise were tabulated. The results are given in Table 1. 
Table 1: Overall LAeq (in $\mathrm{dB} A$ ) and 90th percentile LAeq (in $\mathrm{dB} A$ ) and difference LAeq in dB for an input of $65 \mathrm{~dB}$ SPL for five different types of noise, with NR algorithm 'ON' and NR algorithm 'OFF'.

\begin{tabular}{|c|c|c|c|c|c|c|}
\hline \multirow[b]{2}{*}{ Types of noise } & \multicolumn{2}{|c|}{ NR algorithm 'ON' } & \multicolumn{2}{|c|}{ NR algorithm 'OFF' } & \multicolumn{2}{|c|}{$\begin{array}{c}\text { Difference in LAeq (dB) } \\
\text { with NR Algorithm-on Vs. } \\
\text { NR algorithm-off }\end{array}$} \\
\hline & $\begin{array}{l}\text { Overall LAeq } \\
\text { (dB A) }\end{array}$ & $\begin{array}{c}\text { LAeq } \\
\text { (dB A) } \\
\text { at 90th } \\
\text { percentile }\end{array}$ & $\begin{array}{l}\text { Overall LAeq } \\
\text { (dB A) }\end{array}$ & $\begin{array}{c}\text { LAeq } \\
(\mathrm{dB} A) \\
\text { at } 90 \text { th } \\
\text { percentile }\end{array}$ & Overall LAeq & $\begin{array}{l}\text { LAeq 90th } \\
\text { percentile }\end{array}$ \\
\hline Cafeteria noise & 61.1 & 56.5 & 63.4 & 60.7 & 2.3 & 4.0 \\
\hline Fan noise & 60.1 & 58.0 & 63.3 & 61.5 & 3.1 & 3.5 \\
\hline Speech babble & 62.8 & 58.9 & 63.2 & 60.4 & 0.4 & 1.5 \\
\hline Traffic noise & 63.7 & 51.8 & 64.1 & 59.1 & 0.4 & 7.3 \\
\hline White noise & 59.8 & 59.4 & 64 & 63.6 & 4.2 & 4.2 \\
\hline
\end{tabular}

From Table 1, it can be noted that the mean overall and $90^{\text {th }}$ percentile LAeq values in $\mathrm{dB}(\mathrm{A})$ for NR algorithm 'ON' condition was lower than that for NR algorithm 'OFF' condition, for all types of noise. This difference between the two hearing aid conditions was more in the $90^{\text {th }}$ percentile values. From this, it can be inferred that there is an effective reduction in the level of noise when NR of the hearing aid was enabled.

\section{Speech in Noise Condition}

The hearing aid output for speech in noise condition with $\mathrm{NR}$ algorithm 'ON' and NR algorithm 'OFF' was evaluated using WADA-SNR, PESQ and EDI measures. The mean and standard deviation for WADA-SNR (Table 2), PESQ (Table 3) and EDI (Table 4) for ten sentences, for five types of noise, three input SNRs for NR algorithm 'ON' and NR algorithm 'OFF' conditions have been tabulated. These tables also depict the difference in the mean WADA-SNR, PESQ and EDI values between the NR algorithm 'ON' and 'OFF' conditions.

From Table 2, Table 3 and Table 4 it can be observed that the mean values for WADA-SNR, PESQ and EDI parameters in NR algorithm 'ON' and NR algorithm 'OFF' for $+5,0$ and -5 $\mathrm{dB}$ input SNRs follow a pattern. When the input SNR was high: the signal to noise ratio at the output was also high as revealed by values of WADA-SNR; the output from the hearing aid was more corresponding to the unprocessed signal (clear speech) as depicted by PESQ values, and the envelope of the signal was represented better as shown by EDI values.

In addition, there were differences in mean values for WADASNR, PESQ and EDI parameters at $+5,0$ and $-5 \mathrm{~dB}$ input SNRs between NR algorithm 'ON' and NR algorithm 'OFF' conditions. Also, there is a definite improvement from NR as evident from the difference mean values $(\Delta)$ of WADA-SNR, PESQ and EDI. It can also be seen that with higher input SNR, there was a greater improvement observed from NR. The amount of benefit from NR varied for each type of noise, though the input SNR remained the same, i.e., cafeteria noise at $+5 \mathrm{~dB}$ and fan noise at $+5 \mathrm{~dB}$ do not have similar improvements in SNR at the output of the hearing aid. Similar results were obtained for EDI. The NR algorithm 'ON' condition had lower EDI values compared to NR algorithm 'OFF' condition. The lower EDI values, in NR algorithm 'ON' condition in comparison with unprocessed speech, signify closer resemblance of the processed and unprocessed signal. This was also evident from difference EDI values $(\Delta \mathrm{EDI})$. As seen for WADA-SNR, in the NR algorithm 'ON' condition, the amount of improvement in signal envelope (EDI) varied for each type of noise, at comparable input SNR values. The PESQ values also revealed a pattern that was similar to the values of WADA-SNR and EDI. 


\section{Global Journal of Otolaryngology}

Table 2: Mean, standard deviation and difference values for WADA-SNR, for five types of noise and three input SNRs, in NR algorithm 'ON' and NR algorithm 'OFF' conditions.

\begin{tabular}{|c|c|c|c|c|c|c|}
\hline \multirow{3}{*}{ Types of noise } & \multirow{3}{*}{$\begin{array}{c}\text { Input } \\
\text { SNR }\end{array}$} & \multicolumn{4}{|c|}{ WADA-SNR (dB) } & \multirow{3}{*}{$\begin{array}{c}\text { Difference } \\
\text { between WADA- } \\
\text { SNR } \\
\text { (dB) } \\
\text { NR algorithm- } \\
\text { on Vs. NR } \\
\text { algorithm-off }\end{array}$} \\
\hline & & \multicolumn{2}{|c|}{ NR algorithm 'ON' } & \multicolumn{2}{|c|}{ NR algorithm 'OFF' } & \\
\hline & & Mean & SD & Mean & SD & \\
\hline \multirow{3}{*}{ Cafeteria noise } & +5 & 12.76 & 1.99 & 5.66 & 1.30 & 7.1 \\
\hline & 0 & 8.13 & 2.07 & 1.99 & 1.31 & 6.14 \\
\hline & -5 & 3.57 & 2.20 & -1.25 & 1.45 & 4.82 \\
\hline \multirow{3}{*}{ Fan noise } & +5 & 12.34 & 2.00 & 4.86 & 1.37 & 7.48 \\
\hline & 0 & 6.93 & 1.53 & 0.91 & 1.29 & 6.02 \\
\hline & -5 & 0.44 & 1.80 & -2.83 & 1.26 & 3.27 \\
\hline \multirow{3}{*}{ Speech babble } & +5 & 11.54 & 1.60 & 5.12 & 1.25 & 6.42 \\
\hline & 0 & 3.65 & 2.59 & 1.75 & 1.25 & 1.9 \\
\hline & -5 & 0.73 & 2.70 & -0.70 & 1.00 & 1.43 \\
\hline \multirow{3}{*}{ Traffic Noise } & +5 & 16.09 & 2.29 & 7.78 & 1.64 & 8.31 \\
\hline & 0 & 12.65 & 3.22 & 4.98 & 2.31 & 7.67 \\
\hline & -5 & 9.83 & 3.48 & 2.75 & 3.50 & 7.08 \\
\hline
\end{tabular}

Table 3: Mean, standard deviation and difference values for PESQ, for five types of noise and three input SNRs between NR algorithm 'ON' and NR algorithm 'OFF'.

\begin{tabular}{|c|c|c|c|c|c|c|}
\hline \multirow{3}{*}{ Types of noise } & \multirow{3}{*}{ Input SNR } & \multicolumn{4}{|c|}{ PESQ } & \multirow{3}{*}{$\begin{array}{l}\text { Difference PESQ } \\
\text { between NR } \\
\text { algorithm-on Vs. } \\
\text { NR algorithm-off }\end{array}$} \\
\hline & & \multicolumn{2}{|c|}{ NR algorithm 'ON' } & \multicolumn{2}{|c|}{ NR algorithm 'OFF' } & \\
\hline & & Mean & SD & Mean & SD & \\
\hline \multirow{3}{*}{ Cafeteria noise } & +5 & 2.39 & 0.20 & 2.20 & 0.23 & 0.19 \\
\hline & 0 & 2.03 & 0.23 & 1.87 & 0.34 & 0.16 \\
\hline & -5 & 1.44 & 0.47 & 1.44 & 0.24 & 0 \\
\hline \multirow{3}{*}{ Fan noise } & +5 & 2.33 & 0.21 & 2.17 & 0.24 & 0.16 \\
\hline & 0 & 2.01 & 0.26 & 1.91 & 0.28 & 0.1 \\
\hline & -5 & 1.55 & 0.57 & 1.81 & 0.12 & -0.26 \\
\hline \multirow{3}{*}{ Speech babble } & +5 & 2.25 & 0.21 & 2.18 & 0.21 & 0.07 \\
\hline & 0 & 1.95 & 0.29 & 1.95 & 0.24 & 0 \\
\hline & -5 & 1.30 & 0.51 & 1.47 & 0.40 & -0.17 \\
\hline \multirow{3}{*}{ Traffic Noise } & +5 & 2.60 & 0.33 & 2.51 & 0.22 & 0.09 \\
\hline & 0 & 2.20 & 0.32 & 2.23 & 0.21 & -0.03 \\
\hline & -5 & 1.89 & 0.27 & 1.98 & 0.22 & -0.09 \\
\hline \multirow{3}{*}{ White noise } & +5 & 2.72 & 0.15 & 2.65 & 0.17 & 0.07 \\
\hline & 0 & 2.33 & 0.16 & 2.42 & 0.16 & -0.09 \\
\hline & -5 & 2.04 & 0.19 & 2.22 & 0.15 & -0.18 \\
\hline
\end{tabular}

Note. The range of PESQ value is from 0 to 4.5 ; higher the value, better the perceived quality. 


\section{Global Journal of Otolaryngology}

Table 4: Mean, standard deviation and difference values for EDI for five types of noise and three SNRs in NR algorithm 'ON' and NR algorithm 'OFF', averaged for ten sentences.

\begin{tabular}{|c|c|c|c|c|c|c|}
\hline \multirow{3}{*}{ Types of noise } & \multirow{3}{*}{$\begin{array}{l}\text { Input } \\
\text { SNR }\end{array}$} & \multicolumn{4}{|c|}{ EDI } & \multirow{3}{*}{$\begin{array}{l}\text { Difference in EDI } \\
\text { between NR } \\
\text { algorithm-on } \\
\text { Vs. NR }\end{array}$} \\
\hline & & \multicolumn{2}{|c|}{ NR algorithm 'ON' } & \multicolumn{2}{|c|}{ NR algorithm 'OFF' } & \\
\hline & & Mean & SD & Mean & SD & \\
\hline & & & & & & algorithm-off \\
\hline \multirow{3}{*}{$\begin{array}{l}\text { Cafeteria noise } \\
\text { (CN) }\end{array}$} & +5 & 0.15 & 0.03 & 0.17 & 0.03 & -0.02 \\
\hline & 0 & 0.20 & 0.03 & 0.24 & 0.03 & -0.04 \\
\hline & -5 & 0.27 & 0.03 & 0.29 & 0.03 & -0.02 \\
\hline \multirow{3}{*}{ Fan noise (FN) } & +5 & 0.14 & 0.01 & 0.17 & 0.04 & -0.03 \\
\hline & 0 & 0.18 & 0.03 & 0.24 & 0.04 & -0.06 \\
\hline & -5 & 0.26 & 0.03 & 0.30 & 0.03 & -0.04 \\
\hline \multirow{3}{*}{$\begin{array}{l}\text { Speech babble } \\
\text { (SB) }\end{array}$} & +5 & 0.15 & 0.01 & 0.19 & 0.03 & -0.04 \\
\hline & 0 & 0.24 & 0.03 & 0.25 & 0.03 & -0.01 \\
\hline & -5 & 0.30 & 0.03 & 0.31 & 0.03 & -0.01 \\
\hline \multirow{3}{*}{ Traffic Noise (TN) } & +5 & 0.16 & 0.05 & 0.17 & 0.03 & -0.01 \\
\hline & 0 & 0.22 & 0.06 & 0.24 & 0.02 & -0.02 \\
\hline & -5 & 0.30 & 0.41 & 0.30 & 0.02 & 0 \\
\hline \multirow{3}{*}{ White noise (WN) } & +5 & 0.13 & 0.01 & 0.19 & 0.03 & -0.06 \\
\hline & 0 & 0.18 & 0.03 & 0.26 & 0.03 & -0.08 \\
\hline & -5 & 0.26 & 0.04 & 0.30 & 0.03 & -0.04 \\
\hline
\end{tabular}

Note. The range of EDI value is from 0 to 1 . Higher the value, poorer is the measure.

In order to test if the data on WADA-SNR, EDI and PESQ were normally distributed, Kolmogorov-Smirnov Z-test of normality was done. The results revealed that the data were normally distributed ( $p>0.05$ ) within each noise and SNRs for all parameters across ten sentences. To know whether the trend was significantly different for NR algorithm, type of noise and input SNR for WADA-SNR, PESQ and EDI, averaged for the ten sentences, repeated measures multivariate analysis of variance (MANOVA) was done.

Table 5: Main effects and interaction for NR algorithm, type of noise and input SNR for WADA-SNR, PESQ and EDI, averaged for the ten sentences as revealed on MANOVA.

\begin{tabular}{|c|c|c|c|c|c|c|}
\hline \multirow{2}{*}{$\begin{array}{c}\text { MAIN EFFECT AND } \\
\text { INTERACTION }\end{array}$} & \multicolumn{2}{|c|}{ WADA-SNR } & \multicolumn{2}{c|}{ PESQ } & \multicolumn{2}{c|}{ EDI } \\
\cline { 2 - 7 } & F (df, ERROR) & P & F (df, ERROR) & P & F (df, ERROR) & P \\
\hline NR algorithm & $605.13(1,9)$ & $0.000^{*}$ & $0.002(1,9)$ & 0.962 & $53.33(1,9)$ & $0.000^{*}$ \\
\hline Noise & $28.61(4,36)$ & $0.000^{*}$ & $34.61(4,36)$ & $0.000^{*}$ & $1.80(4,36)$ & 0.20 \\
\hline SNR & $801.81(2,18)$ & $0.000^{*}$ & $241.78(2,18)$ & $0.000^{*}$ & $997.16(2,18)$ & $0.000^{*}$ \\
\hline $\begin{array}{c}\text { NR algorithm* } \\
\text { Noise }\end{array}$ & $44.95(4,36)$ & $0.000^{*}$ & $2.89(4,36)$ & 0.059 & $25.75(4,36)$ & $0.000^{*}$ \\
\hline NRalgorithm* SNR & $54.90(2,18)$ & $0.000^{*}$ & $15.05(2,18)$ & $0.002^{*}$ & $10.92(2,18)$ & $0.005^{* *}$ \\
\hline Noise *SNR & $24.46(8,72)$ & $0.000^{*}$ & $4.39(8,72)$ & $0.031^{* *}$ & $4.53(8,72)$ & $0.009^{* *}$ \\
\hline $\begin{array}{c}\text { NR algorithm* } \\
\text { Noise*SNR }\end{array}$ & $13.88(8,72)$ & $0.000^{*}$ & $0.75(8,72)$ & 0.517 & $5.84(8,72)$ & $0.002^{* *}$ \\
\hline
\end{tabular}

Note. ${ }^{*}$ indicates significance at $0.001(p<0.001) ;{ }^{*}$ indicates significance at $0.05(p<0.05)$.
The results of repeated measures MANOVA revealed a significant main effect across the variables. However, main effect 'ON' and 'OFF') in PESQ parameter and for Noise (across five different types of noise) in EDI parameter. There was an interaction between NR algorithm, noise and three input SNRs for WADA-SNR, PESQ and EDI parameters. Interaction was seen for all the conditions except for NR algorithm *Noise and NR algorithm*Noise*SNR in PESQ parameter. Table 5 gives the main effects and interaction for NR, type of noise and input SNR for WADA-SNR, PESQ and EDI averaged for the ten sentences. was not observed for NR algorithm (between NR algorithm 
To test the main effect and interaction, individual repeated measures analysis of variance (ANOVA) was done for NR algorithm 'ON' and NR algorithm 'OFF' conditions. Though repeated measures MANOVA showed main effect, because there were significant 2-way and 3-way interactions between the variables, post-hoc analysis was not considered. Thus, paired t-test was done to test the difference between NR algorithm 'ON' and NR algorithm 'OFF' conditions, i.e., comparison was done between each type of noise at a particular input SNR, NR algorithm ' $\mathrm{ON}$ ' and with the corresponding type of noise and input SNR, NR algorithm 'OFF'. However, paired t-test was done only for WADA-SNR and EDI parameters between NR algorithm 'ON' and 'OFF' conditions since there was no main effect seen in PESQ parameter for NR algorithm in repeated measures MANOVA.

Table 6: Paired t-test between NR algorithm 'ON' and NR algorithm 'OFF' for WADA-SNR and EDI parameters.

\begin{tabular}{|c|c|c|c|c|c|c|c|}
\hline \multirow{2}{*}{ Input SNR } & \multirow{2}{*}{ Noise } & \multicolumn{3}{|c|}{ WADA-SNR } & \multicolumn{3}{|c|}{ EDI } \\
\hline & & $\mathbf{t}$ & df & $\mathbf{p}$ & $\mathbf{t}$ & df & $\mathbf{p}$ \\
\hline \multirow{5}{*}{ +5 dB SNR } & $\mathrm{CN}$ & 18.934 & 9 & $0.000^{*}$ & -1.981 & 9 & 0.79 \\
\hline & FN & 31.789 & 9 & $0.000^{*}$ & -3.854 & 9 & $0.004^{*}$ \\
\hline & SB & 37.058 & 9 & $0.000^{*}$ & -5.524 & 9 & $0.000^{*}$ \\
\hline & $\mathrm{TN}$ & 27.463 & 9 & $0.000^{*}$ & -1.381 & 9 & 0.201 \\
\hline & WN & 49.590 & 9 & $0.000^{*}$ & -8.272 & 9 & $0.000^{*}$ \\
\hline \multirow{5}{*}{0 dB SNR } & $\mathrm{CN}$ & 19.354 & 9 & $0.000^{*}$ & -7.499 & 9 & $0.000^{*}$ \\
\hline & FN & 28.572 & 9 & $0.000^{*}$ & -11.974 & 9 & $0.000 *$ \\
\hline & SB & 2.567 & 9 & 0.030 & -1.937 & 9 & 0.085 \\
\hline & $\mathrm{TN}$ & 19.076 & 9 & $0.000^{*}$ & -1.469 & 9 & 0.176 \\
\hline & $\mathrm{WN}$ & 27.478 & 9 & $0.000^{*}$ & -30.772 & 9 & $0.000^{*}$ \\
\hline \multirow{5}{*}{$-5 \mathrm{~dB}$ SNR } & $\mathrm{CN}$ & 14.108 & 9 & $0.000^{*}$ & -5.993 & 9 & $0.000^{*}$ \\
\hline & FN & 8.612 & 9 & $0.000^{*}$ & -12.769 & 9 & $0.000^{*}$ \\
\hline & SB & 2.095 & 9 & 0.066 & -2.584 & 9 & 0.030 \\
\hline & $\mathrm{TN}$ & 11.926 & 9 & $0.000^{*}$ & 0.472 & 9 & 0.648 \\
\hline & $\mathrm{WN}$ & 8.504 & 9 & $0.000^{*}$ & -11.185 & 9 & $0.000^{*}$ \\
\hline
\end{tabular}

Note. ${ }^{*}$ significant at 0.01 level calculated according to corrected Bonferroni $(p<0.01)$
A. Comparison of WADA-SNR, PESQ and EDI Parameters across Different Types of Noise and within Input SNRs and Across Different Input SNRs within each Type of Noise for NR algorithm 'OFF' Condition.

Across different types of noise. Repeated measures ANOVA within NR algorithm 'OFF' condition for WADA-SNR, PESQ and EDI across five types of noise (cafeteria, fan, speech babble, traffic and white noise) was done. The results from WADASNR revealed that there was a significant difference between different types of noise at $+5 \mathrm{~dB} \operatorname{SNR}[\mathrm{F}(4,36)=17.56, \mathrm{p}<0.001]$, $0 \mathrm{~dB}$ SNR $[\mathrm{F}(4,36)=23.11, \mathrm{p}<0.001]$ and at $-5 \mathrm{~dB} \operatorname{SNR}[\mathrm{F}(4$,
The paired t-test results indicated that there was a significant difference between NR algorithm 'ON' and 'OFF' conditions for WADA-SNR parameter across all input SNRs and for all types of noise, except for speech babble at $0 \mathrm{~dB}$ SNR and at $-5 \mathrm{~dB}$ SNR. For EDI, there was no significant difference between NR algorithm 'ON' and 'OFF' condition for cafeteria noise at $+5 \mathrm{~dB}$ SNR. It was also observed that there was no significant difference for traffic noise at all input SNRs. At $0 \mathrm{~dB}$ SNR and $-5 \mathrm{~dB}$ SNR, speech babble did not have significant difference in EDI parameter between NR algorithm 'ON' and 'OFF' (Table 6). In order to test if the WADASNR, PESQ and EDI parameters vary between different types of noise and input SNRs, within NR algorithm 'ON' and 'OFF' condition, repeated measures ANOVA was performed i.e., in $\mathrm{NR}$ algorithm 'ON' and 'OFF' conditions, across different types of noise and within input SNRs and across different input SNRs within each type of noise.
$36)=23.19, p<0.001]$. In order to study the types of noise that differed from each other, adjustments for multiple comparisons, Bonferroni post-hoc analysis was done. The results showed that only WADA-SNR of white noise was significantly different from all other types of noise at 0 and -5 dB SNRs. At $+5 \mathrm{~dB}$ SNR, only cafeteria noise and traffic noise were significantly different from white noise.

The results of PESQ showed that there is a significant difference between different types of noise at $+5 \mathrm{~dB}$ SNR [ $\mathrm{F}(4$, $36)=46.70, \mathrm{p}<0.001], 0 \mathrm{~dB} \operatorname{SNR}[\mathrm{F}(4,36)=24.35, \mathrm{p}<0.001]$ and at $-5 \mathrm{~dB}$ SNR $[\mathrm{F}(4,36)=26.39, \mathrm{p}<0.001]$. The results of 
adjustments for multiple comparisons, Bonferroni post-hoc analysis was similar to that of WADA-SNR. The results showed that only white noise was significantly different from all other types of noise at 0 and $-5 \mathrm{~dB}$ SNRs. Also, at $0 \mathrm{~dB}$, traffic noise was significantly different from fan noise and speech babble. At +5 dB SNR, traffic noise was significantly different from cafeteria noise, fan noise and speech babble. In addition, white noise was significantly different from fan noise and speech babble at +5 dB SNR.

It was noted that, there was a significant difference between different types of noise for $\operatorname{EDI}$ at $+5 \mathrm{~dB} \operatorname{SNR}[\mathrm{F}(4,36)=14.29$, $\mathrm{p}<0.01]$. However, there was no significant difference between different types of noise at $0 \mathrm{~dB} \operatorname{SNR}[\mathrm{F}(4,36)=2.02$, p>0.05] and at $-5 \mathrm{~dB}$ SNR $[\mathrm{F}(4,36)=0.58, \mathrm{p}>0.05]$. In order to know the types of noise that differed from each other at $+5 \mathrm{~dB}$ SNR, adjustments for multiple comparisons, Bonferroni post-hoc analysis was done. The results indicated that EDI of fan noise was significantly different from cafeteria noise, speech babble and white noise. In addition, cafeteria noise was significantly different from white noise.

Across different input SNRs $(+5,0$ and $-5 \mathrm{~dB})$ within noise. Repeated measures ANOVA within NR algorithm 'OFF' condition for WADA-SNR, PESQ and EDI parameters across different input SNRs $(+5,0$ and $-5 \mathrm{~dB})$ was done. The results revealed that there was a significant difference across different SNRs for WADA-SNR, PESQ and EDI for all types of noise. The results have been tabulated in Table 7 . In order to study the input SNRs that differed from each other with each type of noise, adjustments for multiple comparisons, Bonferroni post-hoc analysis was done. The results showed that WADA-SNR, PESQ and EDI parameters were significantly different for all types of noise across the all the three SNRs. However, PESQ parameter did not show any significant difference between $0 \mathrm{~dB}$ SNR and -5 dB SNR for fan noise.

Table 7: Repeated measures ANOVA within NR algorithm 'OFF' condition for WADA-SNR, PESQ and EDI parameters across different input SNRs $(+5,0$ and $-5 \mathrm{~dB})$ within each noise.

\begin{tabular}{|c|c|c|c|c|c|c|}
\hline Noise & \multicolumn{2}{|c|}{ WADA- SNR } & \multicolumn{2}{c|}{ PESQ } & \multicolumn{2}{c|}{ EDI } \\
\hline & F (df, error) & p & F (df, error) & p & F (df, error) & p \\
\hline Cafeteria & $1036.56(2,18)$ & $0.000^{*}$ & $29.54(2,18)$ & $0.000^{*}$ & $1400.66(2,18)$ & $0.000^{*}$ \\
\hline Fan & $729.23(2,18)$ & $0.000^{*}$ & $18.67(2,18)$ & $0.000^{*}$ & $546.77(2,18)$ & $0.000^{*}$ \\
\hline Speech Babble & $499.56(2,18)$ & $0.000^{*}$ & $31.20(2,18)$ & $0.000^{*}$ & $1549.82(2,18)$ & $0.000^{*}$ \\
\hline Traffic & $32.02(2,18)$ & $0.000^{*}$ & $260.85(2,18)$ & $0.000^{*}$ & $464.34(2,18)$ & $0.000^{*}$ \\
\hline White noise & $233.63(2,18)$ & $0.000^{*}$ & $196.01(2,18)$ & $0.000^{*}$ & $1763.76(2,18)$ & $0.000^{*}$ \\
\hline
\end{tabular}

Note:* significant at 0.001 level

\section{B. Comparison of WADA-SNR, PESQ and EDI parameters across different types of noise and within input SNRs and across different input SNRs within each type of noise for $\mathrm{NR}$ algorithm ' $\mathrm{ON}$ ' condition.}

Across different types of noise. Repeated measures ANOVA was performed within NR algorithm 'ON' condition for WADASNR, PESQ and EDI across five types of noises (cafeteria, fan, speech babble, traffic and white noise) within each input SNR. Results from WADA-SNR indicated that there was a significant difference between different types of noise at $+5 \mathrm{~dB}$ input SNR $[\mathrm{F}(4,36)=13.69, \mathrm{p}<0.001], 0 \mathrm{~dB}$ input $\operatorname{SNR}[\mathrm{F}(4,36)=27.94$, $\mathrm{p}<0.001]$ and at $-5 \mathrm{~dB}$ input $\operatorname{SNR}[\mathrm{F}(4,36)=47.66, \mathrm{p}<0.001]$. Since there was significant difference, to study the types of noise that differed from each other, adjustments for multiple comparisons, Bonferroni post-hoc analysis was done.

The results showed that WADA-SNR of traffic noise was significantly different from speech babble and white noise at +5 $\mathrm{dB}$ input SNR. At $0 \mathrm{~dB}$ input SNR, white noise was significantly different from cafeteria noise, fan noise and traffic noise. It was also seen that speech babble differed significantly from cafeteria noise. However, at $-5 \mathrm{~dB}$ input SNR, cafeteria noise was significantly different from all noise except speech babble. The traffic noise was significantly different from all other types of noise.

Analysis of data on PESQ demonstrated that there was a significant difference between different types of noise at +5 $\mathrm{dB}$ input SNR $[\mathrm{F}(4,36)=21.03, \mathrm{p}<0.001], 0 \mathrm{~dB}$ input SNR $[\mathrm{F}$ $(4,36)=9.27, \mathrm{p}<0.01]$ and at $-5 \mathrm{~dB}$ input $\operatorname{SNR}[\mathrm{F}(4,36)=7.70$, $\mathrm{p}<0.01]$. Adjustments for multiple comparisons, Bonferroni post-hoc analysis was done to find out the types of noise that differed from each other. The results unveiled that white noise was significantly different from cafeteria noise at all the three input SNRs. In addition, white noise was significantly different from speech babble and fan noise at $+5 \mathrm{~dB}$ input SNR and with only fan noise at $0 \mathrm{~dB}$ input SNR.

On a similar line, EDI showed that there was a significant difference between different types of noise at $0 \mathrm{~dB}$ input SNR [F $(4,36)=5.95, \mathrm{p}<0.05]$ and at $-5 \mathrm{~dB}$ input $\operatorname{SNR}[\mathrm{F}(4,36)=5.06$, $\mathrm{p}<0.05]$. However, there was no significant difference between different types of noise at $+5 \mathrm{~dB}$ input $\operatorname{SNR}[\mathrm{F}(4,36)=1.95$, $p>0.05]$. Bonferroni post-hoc analysis showed that only EDI of white noise was significantly different from speech babble at 0 dB input SNR. 


\section{Global Journal of Otolaryngology}

Table 8: Repeated measures ANOVA within NR algorithm 'ON' condition for WADA-SNR, PESQ and EDI parameters across different input SNRs $(+5,0$ and $-5 \mathrm{~dB})$ within each noise.

\begin{tabular}{|c|c|c|c|c|c|c|}
\hline \multirow{2}{*}{ Noise } & \multicolumn{2}{|c|}{ WADA- SNR } & \multicolumn{2}{c|}{ PESQ } & \multicolumn{2}{c|}{ EDI } \\
\hline & F (df, error) & p & F (df, error) & p & F (df, error) & p \\
\hline Cafeteria & $202.92(2,18)$ & $0.000^{*}$ & $46.64(2,18)$ & $0.000^{*}$ & $164.53(2,18)$ & $0.000^{*}$ \\
\hline Fan & $415.95(2,18)$ & $0.000^{*}$ & $30.62(2,18)$ & $0.000^{*}$ & $161.73(2,18)$ & $0.000^{*}$ \\
\hline Speech Babble & $154.73(2,18)$ & $0.000^{*}$ & $27.43(2,18)$ & $0.000^{*}$ & $262.27(2,18)$ & $0.000^{*}$ \\
\hline Traffic & $49.01(2,18)$ & $0.000^{*}$ & $87.33(2,18)$ & $0.000^{*}$ & $145.96(2,18)$ & $0.000^{*}$ \\
\hline White noise & $1419.48(2,18)$ & $0.000^{*}$ & $190.53(2,18)$ & $0.000^{*}$ & $184.22(2,18)$ & $0.000^{*}$ \\
\hline
\end{tabular}

Note:* significant at 0.001 level

Across different input SNRs (+5, 0 and -5 dB) within noise. Repeated measures ANOVA within NR algorithm 'ON' condition for WADA-SNR, PESQ and EDI parameters across different input SNRs $(+5,0$ and $-5 \mathrm{~dB})$ demonstrated that there was a significant difference across different SNRs for WADA-SNR, PESQ and EDI for all types of noise, similar to results of NR algorithm 'OFF' condition. The results have been tabulated in Table 8. Bonferroni post-hoc analysis indicated that WADA-SNR, PESQ and EDI parameters were significantly different for all types of noise across all the three input SNRs.

\section{Discussion}

\section{Noise Alone Condition}

The results from noise alone condition revealed that for NR algorithm 'ON' condition, the overall and 90th percentile LAeq were lower than for NR algorithm 'OFF' condition for all types of noise. However, this difference was marked at the 90th percentile. From this it can be construed that when NR algorithm was ' $\mathrm{ON}^{\prime}$ ' in the hearing aid, it would aid in reducing the noise. Depending on the spectral characteristics of noise, it was observed that steady noise (cafeteria, fan \& white noise) had greater reduction when compared to noise that had more speech-like varying spectrum viz., speech babble. Traffic noise had greater reduction at 90th percentile in NR algorithm 'ON' (51.8 dB) compared to NR algorithm 'OFF' (59.1 dB) i.e., 7.3 $\mathrm{dB}$ reduction, which could be attributed to its unique temporal characteristics i.e., impulsive nature at irregular intervals and decreased modulations.

In a similar study, Summe [39] found that there was more reduction in noise for some of the types of noise viz., jet noise and fan noise by $4.6 \mathrm{~dB}$ and $2.4 \mathrm{~dB}$ respectively, with NR algorithm 'ON' and only for a few hearing aids. The effectiveness of NR algorithm was studied by Rout et al. [13] with 20 new stimuli of which speech-shaped steady state noise provided an average reduction in noise varying from $2.5 \mathrm{~dB}$ to $13 \mathrm{~dB}$ across hearing aids. Zakis et al. [40] studied the effects of four configurations of an environmental noise reduction (ENR) algorithm on preferences, speech understanding and satisfaction. They reported that the gain reduction at $0 \mathrm{~dB}$ modulation depth was either $10 \mathrm{~dB}$ in all channels (ENR strong flat) or shaped from 2-10 dB across channels according to a speech importance function. It could be concluded that modulation based NR algorithm when ' $\mathrm{N}^{\prime}$ would aid in reducing the noise.

\section{Speech in Noise Condition}

The paired t-test results indicated that there was a significant difference between NR algorithms 'ON' and 'OFF' conditions for WADA-SNR parameter across all input SNRs and for all types of noise, which would indicate that NR algorithm was helpful in reducing noise. However, there was no significant difference for speech babble at $0 \mathrm{~dB}$ input SNR and at $-5 \mathrm{~dB}$ input SNR. This could be due to the fact that the other types of noise had nonspeech like characteristics and speech babble had speech-like characteristics. Clean speech has considerably more amplitude fluctuations than speech in a babble or speech-shaped noise [41], hence making a modulation based algorithm difficult in differentiating speech and noise.

However, since the spectral and temporal features are better represented for speech at $+5 \mathrm{~dB}$, it could be postulated that noise reduction algorithm could easily differentiate between speech babble and actual speech and thus there was an improvement in output SNR for speech babble only at $+5 \mathrm{~dB}$ of input SNR. From this it can be inferred that with good / favorable SNRs, NR algorithm is able to provide better results for even speech shaped noise. Bentler and Chiou [41] suggest that the signal-tonoise ratio would also influence the trigger of noise reduction algorithm and influence the gain reduction.

For EDI, there was a significant difference between NR algorithm 'ON' and 'OFF' conditions for fan, traffic and white noise at all input SNRs which shows that NR algorithm was capable of reducing noise, in turn depicting a better speech envelope in comparison to NR algorithm 'OFF'. Walaszek [42] evaluated EDI using Danish sentences in the presence of a single female talker background noise or in presence of International Collegium of Rehabilitative Audiology (ICRA) noise. The digital noise reduction and directional microphone modes were disabled. Mean EDIs varied from .12 to .22 depending type of background noise. The present study also reports similar EDI results. The EDI values varied as the background noise changed and across different SNRs and mean EDIs ranged between 0.17 
and 0.30 in the NR algorithm 'OFF'. And EDI ranged from 0.13 to 0.27 in the NR algorithm 'ON' position as the background noise varied across different SNRs.

It was also observed that as the background noise was more stationary, the EDIs were lower than for fluctuating types of noise. Hence, it could be postulated that EDI may aid as a tool to assess temporal variations for a stationary noise like white noise and fan noise and may not be a good tool for assessing highly fluctuating noises. The perceived quality of speech as measured from PESQ parameter showed a better quality representation as the SNR increased. Huber et al. [43] evaluated the performance of objective speech and audio quality measures in predicting the perceived quality of frequency compressed speech in hearing aids. They concluded that there was a very clear association between SNR and PESQ values.

The major difference in SNR values were reflected in the speech quality; a higher SNR (signal to noise ratio) gave a higher PESQ value (speech quality measurement). The PESQ results of the present study are also in congruent with the above. Beerends et al. [32] and Ma et al., [31] have tested the PESQ measure in predicting speech intelligibility. Both studies have reported high correlation ( $r=0.9 ; r=0.77-0.79$ ) of PESQ measure in predicting intelligibility of consonants and sentences in noise. In the present study, it was seen that the speech quality measure was better in the presence of white noise as against all the other types of noise. This could be attributed to the spectral characteristics of the noise and since the NR algorithm in the study was modulation based, NR algorithm was effective in distinguishing speech and noise. Hu and Loizou [19] showed that some noise-suppression algorithms may degrade speech intelligibility by attenuating the spectral characteristics near format frequency in noisy conditions. This may also be the reason that the speech quality measure was not uniform across all types of noise.

In addition, the variation across the parameters WADA-SNR, PESQ and EDI for different types of noise within each input SNR for were not the same since each of these parameters measure a different aspect or feature of the speech signal. As the SNR increased, the speech signal is better represented and vice versa when the SNR decreases. Thus, there were differences observed for each type of noise across SNRs.

Chong and Jenstad [44] assessed the amount of noise level reduction and processing of phonemes by the digital noise reduction in the hearing aids in. Three types of noise were used as stimuli. They found that most of the noise reduction used in hearing aids were effective in reducing only pink and cafeteria noise. However, the ICRA noise was slightly increased. The noise reduction was greater as the number of channels increased. The level of the fricatives did not change after it was processed through DNR, but levels of some fricatives were enhanced up by $10 \mathrm{~dB}$ particularly for pink noise. Similar results was also seen in the present study i.e., it was noted that there was greater reduction of noise in the higher frequencies in the presence of white noise. From the above results, Miller [12] postulated that noise reduction algorithms may potentially improve the SNR.

In the present study, as there was improvement in the output SNR, there was better quality depiction of the output signal and good envelope representation with increase in input SNR. This is evident in the mean values provided across Tables 2-4. The NR algorithm ' $O N$ ' condition had better performance than NR algorithm 'OFF' condition. The action of the noise reduction algorithm might have played a role in identifying the modulations of each type of noise and reducing each type of noise in NR algorithm 'ON' condition. Hence, the combination of both the factors have added in bringing about differences across noise within each input SNR.

\section{Conclusion}

The present study evaluated the effect of noise type and input SNR on the NR algorithm in the hearing aid. Also, the present study utilized parameters which could extract the spectral and temporal aspects of speech signal in assessing the output from hearing aid. This might help in elaborating and understanding the subtle changes in the output of the hearing aid with and without NR algorithm processing. To summarize the findings of the study: NR algorithm in the study was effective in reducing different types of noise encountered daily in the environment as evident from the noise only condition. This may help the hearing aid user to be comfortable in noisy environment. It was also seen that, the effectiveness of NR algorithm varied on temporal and spectral parameters of noise.

As evidenced from previous literature, the present study also concludes that noise types which have more speech-like features are less detected by NR algorithm, than noise that are non-speech like. The effectiveness of NR algorithm also depends on the SNR of the input signal. Higher the SNR of the input signal $(+5 \mathrm{~dB})$, more benefits are derived from NR algorithm. Analyses of the output of the hearing with $\mathrm{NR}$ algorithm ' $\mathrm{ON}$ ' and 'OFF' using parameters WADA-SNR, PESQ and EDI facilitated in concluding that NR algorithm was effective in enhancing certain acoustic parameters of speech such as SNR and envelope; however, the perceptual quality of speech, predicted for acoustical signal was not improved.

Thus, it can be concluded that NR algorithm when 'ON' is helpful in reducing noise which in turn can improve the duration of hearing aid usage and speech perception. However, the amount of reduction provided by NR algorithm depends on the type of noise, and the SNR in the environment. The relation between changes in the objective parameters WADA-SNR, EDI, and PESQ and behavioral measures should be explored. Also, though improvements were derived when NR algorithm was ' $O N$ ' through acoustical measurements, equivalent improvements/ benefits may or may not be reflected in behavioral measures. 
Hence, it is important to verify whether changes in the acoustical signal with $\mathrm{NR}$ algorithm 'ON' are being reflected in the behavioral measure and this needs to be studied to conclude the actual real world benefit of NR algorithm.

\section{Acknowledgement}

We like to acknowledge the Director, All India Institute of Speech and Hearing (AIISH), Mysuru and HOD-Audiology, AIISH, Mysuru for providing the infrastructure and equipment for the study.

\section{References}

1. Bentler RA (2005) Effectiveness of Directional Microphones and Noise Reduction Schemes in Hearing Aids: A Systematic Review of the Evidence. J Am AcadAudiol 16(7): 473-484.

2. Powers TA, Branda E, Hernandez A, Pool A (2006) Study finds real-world benefit from digital noise reduction. Hear J 59(2): 26-30.

3. Oliveira JR, Lopes ES, Alves AF (2010) Speech perception of hearing impaired people using a hearing aid with noise suppression algorithms. Braz J Otorhinolaryngol 76(1): 14-17.

4.Walden BE, Surr RK, Cord MT, Edwards B, Olson L (2000) Comparison of Benefits Provided by Different Hearing Aid Technologies. J Am AcadAudiol 11(10): 540-560.

5. Bentler R, Wu YH, Kettel J, Hurtig R (2008) Digital noise reduction: outcomes from laboratory and field studies. Int J Audiol 47(8): 447-60.

6. Hickson L, Thyer N, Bates D (1999) Acoustic Analysis of Speech through a Hearing Aid: Consonant-Vowel Ratio Effects with Two- Channel Compression Amplification. J Am AcadAudiol 10(10): 549-556.

7. Lai Y, Tang S, Tsai K, Young S (2013) Objective Measurement of Speech Quality for Hearing Aids. J Med Bio Eng 33: 576-582.

8. Peeters H, Kuk F, Lau C, Keenan D (2009) Subjective and Objective Evaluation of Noise Management Algorithms. J Am AcadAudiol 20(2): 89-98.

9. Shiran N, Shallom ID (2009) Enhanced PESQ algorithm for objective assessment of speech quality at a continuous varying delay. Quality of Multimedia Experience, International workshop on, IEEE, San Diego, CA, USA.

10. Zakis JA, Wise C (2007) The acoustic and perceptual effects of two noise-suppression algorithms. J Acoust Soc Am 121(1): 433-441.

11. Souza PE, Tremblay KL (2006) New Perspectives on Assessing Amplification Effects, Trends Amplif 10: 119-143.

12. Miller CW (2013) The Role of Aided Signal-to-Noise Ratio in Aided Speech Perception in Noise Washington: Washington University, USA, (Unpublished doctoral dissertation).

13. Rout A, Hanline LE, Halling DC (2007) New stimuli for evaluation of multichannel noise reduction hearing aids. Proceedings of the European Federation of Audiological Societies from the $8^{\text {th }}$ EFAS Congress / $10^{\text {th }}$ Congress of the German Society of Audiology, Heidelberg, Germany, Europe.

14. Mueller HG, Weber J, Hornsby BWY (2006) The effects of digital noise reduction on the acceptance of background noise. Trends Amplif 10: 83-93.

15. Palmer CV, Bentler R, Mueller HG (2006) Amplification with digital noise reduction and the perception of annoying and aversive sounds. Trends Amplif 10(2): 95-104.
16. Hagerman B, Olofsson A (2004) A method to measure the effect of noise reduction algorithms using simultaneous speech and noise. Acta Acustica 90(2): 356-361.

17. Souza P, Jenstad L, Boike K (2006) Measuring the acoustic effects of compression amplification on speech in noise. J AcoustSoc Am 119(1): 41-44.

18. Naylor G, Johannesson RB (2009) Long-term signal-to-noise ratio at the input and output of amplitude-compression systems. J Am AcadAudiol 20(3): 161-171.

19. Hu Y, Loizou PC (2008) Evaluation of Objective Quality Measures for Speech Enhancement. IEEE Trans. Audio, Speech, and Lang Processing 16(1): 229-238.

20. Eneman K (2008) Evaluation of signal enhancement algorithms for hearing instruments. Presented at the $16^{\text {th }}$ European Signal Processing Conference, Lausanne.

21. Nilsson MJ, Bray VH (2004) The Noise Reduction Index: Benchtop Estimate of SNR Changes. Poster presented at the IHCON, Tahoe City, California, USA.

22. Kressner AA, Anderson DV, Rozell CJ (2012) Evaluating the generalization of the Hearing Aid Speech Quality Index (HASQI). IEEE Trans Audio, Speech, and Lang Proc 407-415.

23. Kim C, Stern RM, International Speech Communication Association (2008) Robust Signal-to-Noise Ratio Estimation Based on Waveform Amplitude Distribution Analysis, Brisbane, Australia, 22-26.

24. Souza P, Hoover E, Gallun F (2012) Application of the Envelope Difference Index to spectrally sparse speech. J Speech Lang Hear Res 55(3): 824-837.

25. Moore BCJ, Glasberg BR, Baer T (1997) A Model for the Prediction of Thresholds, Loudness, and Partial Loudness. J Audio Eng Soc 45(4): 224-240.

26. Kates JM (1985) A central spectrum model for the perception of coloration in filtered Gaussian noise. J Acoust Soc Am 77(4): 1529.

27. Huber R, Kollmeier B (2006) PEMO-Q: A new method for objective audio quality assessment using a model of auditory perception. IEEE Trans Audio, Speech, and Lang Processing 14(6): 1902-1911.

28. Rix AW, Beerends JG, Hollier MP, Hekstra AP (2001) Perceptual evaluation of speech quality (PESQ) - A new method for speech quality assessment of telephone networks and codecs. Acoustics, Speech, and Signal Processing, 2001 IEEE International Conference on, 749-752.

29. Rix AW, Hollier MP (2000) The perceptual analysis measurement system for robust end-to-end speech quality assessment. In Acoustics, Speech, and Signal Processing, 2000 IEEE International Conference on, 1515-1518.

30. Beerends J, Hekstra A, Rix A, Hollier M (2002) Perceptual evaluation of speech quality (PESQ) - The new ITU standard for end-to-end speech quality assessment. Part II - Psychoacoustic model. J Audio Eng Soc 50(10): 765-778

31. Ma J, Hu Y, Loizou PC (2009) Objective measures for predicting speech intelligibility in noisy conditions based on new band-importance functions. J Acoust Soc Am 125(5): 3387-3405.

32. Beerends JG, van Wijngaarden S, van Buuren R (2005) Extension of ITU-T Recommendation P.862 PESQ towards Measuring Speech Intelligibility with Vocoders. In New Directions for Improving Audio Effectiveness (pp. 10-1 - 10-6). Meeting Proceedings RTO-MPHFM-123, Paper 10. Neuilly-sur-Seine, France: RTO.

33. Kates JM, Arehart KH (2010) The Hearing-Aid Speech Quality Index (HASQI). J Audio Eng Soc 58: 363-381. 
34. Geetha C, Kumar SKS, Manjula P (2014) Development and standardization of the sentence identification test in the Kannada language. J Hg Sci 4(1): 0A18-26.

35. Anitha R, Manjula P (2003) Effect of multi-speaker babble in different languages on speech recognition scores in Kannada. Mysuru: All India Institute of Speech and Hearing, (Unpublished Independent project submitted to University of Mysuru).

36. Lanman (2006) Math code for Objective measures of speech quality/ SNR. MATLAB Code.

37. Ellis D (2011) Math code for Objective measures of speech quality/ SNR. MATLAB Code.

38. Fortune TW, Woodruff BD, Preves DA (1994) A new technique for quantifying temporal envelope contrasts. Ear Hear 15(1): 93-99.

39. Summe L (2003) Noise reduction in digital hearing aids with a background of environmental sounds Cincinnati: University of Cincinnati, Unpublished M.A. thesis).
40.Zakis JA, Hau J, Blamey PJ (2009) Environmental noise reduction configuration - Effects on preferences, satisfaction, and speech understanding. Int J Audiol 48(12): 853-867.

41. Bentler RA, Chiou (2006) Digital Noise Reduction: An Overview. Trends Amplif 10(2): 67-82.

42. Walaszek J (2012) Effects of compression in hearing aids on the envelope of the speech signal, Signal based measures of the sideeffects of the compression and their relation to speech intelligibility. Denmark: Technical University of Denmark; 2008. (Unpublished master's thesis). Cited in Souza P, Hoover E, Gallun F. Application of the Envelope Difference Index to spectrally sparse speech. J Speech Lang Hear Res 55(3): 824-837.

43. Huber R, Parsa V, Scollie S (2014) Predicting the perceived sound quality of frequency-compressed speech. PLos One 9(11): e11-26.

44. Chong FY, Jenstad LM (2010) The effects of digital noise reduction algorithms on speech and noise. Poster presented at the International hearing Aid Research Conference, Lake Tahoe, CA, USA.

\section{Your next submission with Juniper Publishers} will reach you the below assets

- Quality Editorial service

- Swift Peer Review

- Reprints availability

- E-prints Service

- Manuscript Podcast for convenient understanding

- Global attainment for your research

- Manuscript accessibility in different formats

( Pdf, E-pub, Full Text, Audio)

- Unceasing customer service

Track the below URL for one-step submission https://juniperpublishers.com/online-submission.php 